\title{
DISEASE WATCH | IN THE NEWS
}

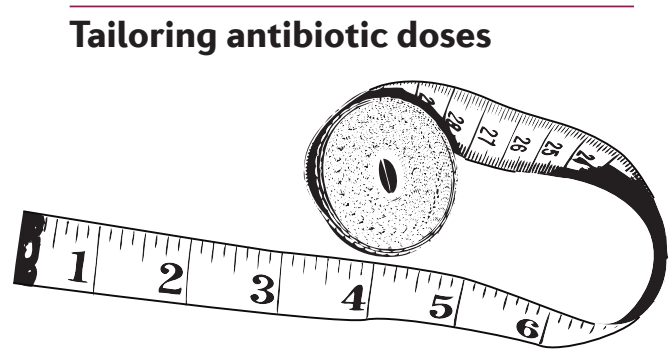

\section{More funding for vaccination}

Bill and Melinda Gates have pledged US $\$ 10$ million over the next 10 years for vaccine research and delivery to the world's poorest countries; this is in addition to the $\$ 4.5$ million donated by the Bill and Melinda Gates Foundation over the past 5 years. The announcement was made at the World Economic Forum meeting in Davos, Switzerland, on the 10-year anniversary of the launch of the GAVI Alliance vaccine consortium. The foundation has predicted that increased vaccination coverage in developing countries could prevent the deaths of 7.6 million children under 5 years of age between 2010 and 2019. If a malaria vaccine can be produced by 2014 , the lives of an additional 1 million children could be saved. Bill Gates urged governments and the private sector to contribute as well. "We must make this the decade of vaccines," he said. "Vaccines already save and improve millions of lives in developing countries. Innovation will make it possible to save more children than ever before." The Times/Reuters could be carried out by an algorithm, as is the current practice for children, who receive antibiotic doses in liquid forms according to their weight. However, because at the moment most antibiotics come in one 'size', a wider range of pill strengths would have to become available, as highlighted in the accompanying editorial. BBC/Lancet

\section{HPV screening for cervical cancer}

Screening for human papilloma virus (HPV) could reduce the risk of cervical cancer in women over 35 years of age, according to a recent study. The authors followed women in Italy between the ages of 25 and 60 who received either a normal cytological test or HPV screening. They found that HPV screening was more effective at detecting pre-cancerous changes in cervical cells early on, thereby providing a longer low-risk period for the women to receive treatment. Although they suggest that HPV screening could be a more effective alternative to smear tests, the authors believe that this strategy would not be suitable for women under 35 years of age, as it may result in diagnosis and unnecessary treatment of problems that would regress naturally. The findings have been welcomed by cancer charities and the UK National Health Service (NHS), which has in fact been testing the use of HPV screening in conjunction with conventional smear tests for cervical cancer diagnosis. BBC/Lancet Oncol.

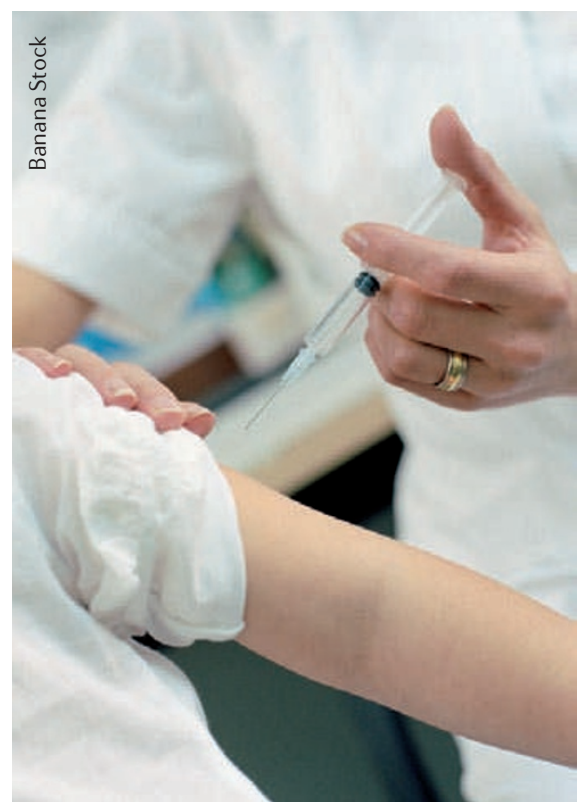

$\overline{\text { New hope for malaria treatments }}$

Two new studies further our chances of producing effective drugs against malaria, a disease that affects millions of people worldwide each year. Bowles and colleagues sequenced the genome of the plant Artemisia annua, from which the antimalarial drug artemisinin is derived, to identify genes and markers that might aid accelerated breeding. Insights from their studies could help breeders to produce varieties of the plant that are bigger and have more leaves, which would facilitate the extraction of artemisin, thereby coping with global demand for the drug. In a separate study in Nature Genetics, Su and colleagues carried out the first genome-wide association study of Plasmodium falciparum from different continents to detect genes that are under selective pressure by antimalarial drugs and that could therefore be associated with the parasite's drug response. Among those identified in this study were genes encoding proteins that are drug or immune targets, such as a membrane antigen and an ATP-binding cassette (ABC) transporter. This study paves the way for a detailed investigation of the changes in the genome of the parasite, which should help to develop more effective treatments. Nature Genetics/Science/The Times

\section{Outbreak news}

$Q$ fever. Thousands of goats have been culled in the Netherlands in an effort to prevent further spread of $\mathrm{Q}$ fever. The disease is caused by Coxiella burnetii, an intracellular bacterium that infects animals and leads to stillbirths, the placentae of which contain billions of bacteria that spread to the environment. In the past 3 years there have been many reported human cases of $\mathrm{Q}$ fever, primarily resulting in pneumonia, and in 2009 alone there were more than 2,300 cases, including 6 deaths. It is still unclear what has caused this outbreak, but experts speculate that it could be the result of intensive goat farming in such a densely populated country or of the emergence of a more virulent strain of the bacterium. An animal vaccine has been developed that prevents the stillbirths, thus blocking the spread of disease, but not infection itself. Although another increase in cases of human $\mathrm{Q}$ fever is expected in the spring of 2010, experts hope that animal vaccination in combination with the culling of all pregnant goats in affected areas will result in reduced numbers of human infections. Science

In the News was compiled with the assistance of David Ojcius, University of California, Merced, USA. David's links to infectious disease news stories can be accessed on Connotea (http://www.connotea. org), under the username NatureRevMicrobiol. Corrected online 16 February 2010. 